Journal for ImmunoTherapy of Cancer

\title{
Tumor copy-number alterations predict response to immune-checkpoint- blockade in gastrointestinal cancer
}

\author{
Zhihao Lu, ${ }^{1}$ Huan Chen, ${ }^{2}$ Shuang Li, ${ }^{1}$ Jifang Gong, ${ }^{1}$ Jian Li, ${ }^{1}$ Jianling Zou, ${ }^{1}$ \\ Lihong Wu, ${ }^{2}$ Jianing $\mathrm{Yu},{ }^{2}$ Wenbo Han, ${ }^{2}$ Huaibo Sun, ${ }^{2} \mathrm{Xi}$ Jiao, ${ }^{1}$ Xiaotian Zhang, ${ }^{1}$ \\ Zhi Peng, ${ }^{1}$ Ming Lu, ${ }^{1}$ Zhenghang Wang, ${ }^{1}$ Henghui Zhang, ${ }^{3}$ Lin Shen (i) ${ }^{1}$
}

To cite: Lu Z, Chen H, Li S, et al. Tumor copy-number alterations predict response to immune-checkpoint-blockade in gastrointestinal cancer. Journal for ImmunoTherapy of Cancer 2020;8:e000374. doi:10.1136/ jitc-2019-000374

- Additional material is published online only. To view please visit the journal online (http://dx.doi.org/10.1136/jitc2019-000374).

ZL, HC and SL contributed equally.

Accepted 05 June 2020

Check for updates

(c) Author(s) (or their employer(s)) 2020. Re-use permitted under CC BY-NC. No commercial re-use. See rights and permissions. Published by BMJ.

${ }^{1}$ Department of Gastrointestinal Oncology, Key laboratory of Carcinogenesis and Translational Research (Ministry of Education), Peking University Cancer Hospital \& Institute,

Beijing, China

${ }^{2}$ Genecast Precision Medicine Technology Institute, Beijing,

China

${ }^{3}$ Institute of Infectious Diseases, Beijing Ditan Hospital, Capital Medical University, Beijing, China

Correspondence to

Lin Shen; shenlin@bjmu.edu.cn

Henghui Zhang;

zhhbao@ccmu.edu.cn

\section{ABSTRACT}

Background Despite the great achievements made in immune-checkpoint-blockade (ICB) in cancer therapy, there are no effective predictive biomarkers in gastrointestinal (Gl) cancer.

Methods This study included 93 metastatic Gl patients treated with ICBs. The first cohort comprising $73 \mathrm{Gl}$ cancer patients were randomly assigned into discovery $(n=44)$ and validation $(n=29)$ cohorts. Comprehensive genomic profiling was performed on all samples to determine tumor mutational burden (TMB) and copy-number alterations (CNAs). A subset of samples was collected for RNA immune oncology (I0) panel sequencing, microsatellite instability (MSI)/mismatch repair and program death ligand 1 (PD-L1) expression evaluation. In addition, 20 gastric cancer $(\mathrm{GC})$ patients were recruited as the second validation cohort.

Results In the first cohort of $73 \mathrm{Gl}$ cancer patients, a lower burden of CNA was observed in patients with durable clinical benefit (DCB). In both the discovery $(n=44)$ and validation $(n=29)$ subsets, lower burden of CNA was associated with an improved clinical benefit and better overall survival (OS). Efficacy also correlated with a higher TMB. Of note, a combinatorial biomarker of TMB and CNA may better stratify DCB patients from ICB treatment, which was further confirmed in the second validation cohort of $20 \mathrm{GC}$ patients. Finally, patients with lower burden of CNA revealed increased immune signatures in our cohort and The Cancer Genome Atlas data sets as well.

Conclusions Our results suggest that the burden of CNA may have superior predictive value compared with other signatures, including PD-L1, MSI and TMB. The joint biomarker of CNA burden and TMB may better stratify DCB patients, thereby providing a rational choice for Gl patients treated with ICBs.

\section{INTRODUCTION}

Encouraged by the great achievements in other cancer types, many ongoing clinical trials are assessing immune-checkpointblockade (ICB) therapy in gastrointestinal (GI) cancers. However, the efficacy is still not satisfactory. ${ }^{1}$ Recently, mounting evidence has tried to identify molecular features relevant to immune responses. ${ }^{2}$ However, data are limited regarding predictive biomarkers in GI cancer patients undergoing ICB treatments.

In particular, microsatellite instability high (MSI-H)/mismatch repair deficient (dMMR) identifies only a small subset $(0 \sim 5 \%)$ of patients with GI cancers. ${ }^{3-5}$ Efficacy analysis to determine the correlation between tumor mutational burden (TMB) and response to ICBs in GI cancers is still underway due to the high variance in TMB within tumor types. ${ }^{6}$ Most recently, evidence has pointed to the predictive role of copy-number alteration (CNA) and immune-related gene expression profile in cancer patients treated with ICB. ${ }^{8-13}$ In specific, a lower burden of copy-number loss (CNloss) was observed in responders to ICB treatment in melanoma. ${ }^{8}$ A combined somatic CNA (SCNA) and TMB score was a candidate biomarker for clinical benefit in patients with melanoma treated with anticytotoxic T-lymphocyte-associated protein 4 (CTLA-4). ${ }^{11}$ Similarly, SCNA was lower in patients with a partial response (PR) than those with progressive disease (PD)/ stable disease $(\mathrm{SD})$ in a cohort of advanced non-small cell lung cancer (NSCLC) patients receiving ICB treatment. ${ }^{14}$ Recently, a negative correlation between CNA and immune parameters including immune cell infiltration and program death ligand 1 (PD-L1) expression was identified in the gastric cancer (GC) and colorectal cancer (CRC) in the The Cancer Genome Atlas (TCGA) data sets. ${ }^{15}$ However, additional insights are still needed for an in-depth view of the burden of CNAs, including copy-number gain (CNgain) and/ or CNloss, in GI cancer patients who received ICB treatments.

Here, we examined tumor specimens from 93 GI cancer patients who were treated with ICBs, including anti-programmed cell death 1 (PD-1), anti-PD-L1, and anti-CTLA-4 antibodies. The predictive and prognostic 
significance of tumor CNA burden was extensively investigated, as well as other biomarkers, including TMB, MSI, and PD-L1.

\section{METHODS}

\section{Patients and study design}

In the first cohort, data of 73 GI cancer patients were retrospectively collected from all GI cancer patients treated with ICBs in the Department of GI Oncology, Peking University Cancer Hospital and Institute from August 1, 2015, to June 8, 2018. To explore genomic correlates of therapeutic efficacy, the 73 GI cancer patients were randomly assigned into the discovery $(n=44)$ and validation ( $n=29)$ GI cohorts. Briefly, formalin-fixed paraffinembedded (FFPE) samples from the 73 patients were subjected to whole-exome sequencing (WES) analysis. To characterize the different immunological features between CNA low and high GI patients, FFPE specimens from a subset of these GI patients $(n=65)$ were subjected to an RNA IO panel sequencing (online supplementary materials).

In addition, 20 patients with advanced GC who were treated with ICBs between January 31, 2018 and May 24, 2019 in the Department of GI Oncology, Peking University Cancer Hospital and Institute were included in this study as an independent GC validation cohort (online supplementary table S1). Aiming to validate the predictive value of CNA burden, we performed WES analysis on these 20 GC samples.

Tumor burden was measured by imaging studies or physical examinations according to the Response Evaluation Criteria in Solid Tumors (RECIST) V.1.1 and iRECIST. Patients were stratified by clinical response. Briefly, the durable clinical benefit (DCB) group was defined as complete response, $\mathrm{PR}$, and SD lasting for $\geq 24$ weeks. No durable benefit (NDB) included patients with PD or SD that lasted $<24$ weeks. ${ }^{16}$

\section{TMB evaluation}

For the determination of TMB value, the number of somatic non-synonymous single nucleotide variants (SNVs) detected using next-generation sequencing was quantified, and the values were extrapolated to the WES analysis using a validated algorithm. ${ }^{17} \mathrm{TMB}$ was measured in mutations per $\mathrm{Mb}$.

\section{Copy-number analysis}

For copy-number analysis, blood cell samples from patients were used as paired controls, and the CONTRA assay was used to call copy-number variations from the FFPE tumor samples for each patient. ${ }^{18}$ CNA burden analysis included measurements of the total CNA burden, CNgain, and CNloss. CNgain/CNloss was defined as the total number of genes with CNgain/CNloss per sample, as previously described. ${ }^{8}$ The CNA burden was calculated as the total number of genes with CNgains/CNlosses. ${ }^{15}$

\section{RNA IO panel sequencing}

RNA IO panel sequencing was conducted as previously described. ${ }^{19}$ Briefly, $10 \mathrm{ng}$ of RNA that was extracted from the FFPE sample was reverse transcribed into cDNA, amplified with a primer pool, and ligated to unique barcode adapters. After purification, the libraries were quantified, pooled, and sequenced on the Ion S5 530 chip (Thermo Fisher Scientific, Waltham, MA). The detailed process of gene expression normalization and measurement of normalized reads per million (nRPM) values are described in the online supplementary materials.

\section{TCGA data sets}

CNA and gene expression data of stomach adenocarcinoma (STAD) and colon adenocarcinoma (COAD) types analyzed in TCGA project were obtained from the cBioPortal (http:/ / www.cbioportal.org). The CNA burden was calculated as the total number of genes with CNgains or CNlosses. ${ }^{15}$ Gene expression levels of the IFN- $\gamma$ signature and expanded immune signature (gene list was provided in the online supplementary materials) were calculated by using the RNA-Seq data. To quantify the relative infiltration of immune cell types into the tumor microenvironment (TME), single-sample gene set enrichment analysis (ssGSEA) was employed as previously reported. ${ }^{20-22}$

\section{Statistical analyses}

Statistical analyses were performed using SPSS V.22.0 software. Statistical tests included Fisher's exact tests, MannWhitney U tests, and Student's t-test. All statistical tests were two-sided.

\section{RESULTS}

\section{Patient characteristics}

This study included 93 metastatic GI patients treated with ICBs between August 1, 2015 and June 8, 2018 ( $\mathrm{n}=73$, comprising discovery and first validation GI cohorts, median follow-up time, 11.97 months, IQR: 6.03-17.3) and between January 31, 2018 and May 24, 2019 ( $\mathrm{n}=20$, second validation GC cohort, median follow-up time, 4.87 months, IQR: 2.83-10.47). To comprehensively explore the genomic correlates with immunotherapy efficacy, 73GI patients were randomly assigned into discovery $(n=44)$ and validation $(n=29)$ cohorts. The baseline and treatment characteristics of all patients are shown in online supplementary table S1. Briefly, of 73 included GI patients, the cancer types were GC (50.7\%), CRC $(27.4 \%)$, and others types, including pancreatic neuroendocrine tumors, GI-NETs, and cholangiocellular carcinoma. In GI combined cohort, 47 patients $(64.4 \%)$ were treated with anti-PD-1 therapy, 15 cases $(20.5 \%)$ were treated with anti-PD-L1 therapy, and 11 cases (15.1\%) received ICB combinational therapy. In the second validation GC cohort $(n=20)$, the cancer types were GC (100\%). Among the GC cases, 15 (75\%) patients were treated with anti-PD-1 therapy, 3 cases $(15 \%)$ were treated with anti-PD-L1 therapy, and 2 cases $(10 \%)$ were treated with ICB combinational therapy (online supplementary table $\mathrm{S} 1$ ). 
A
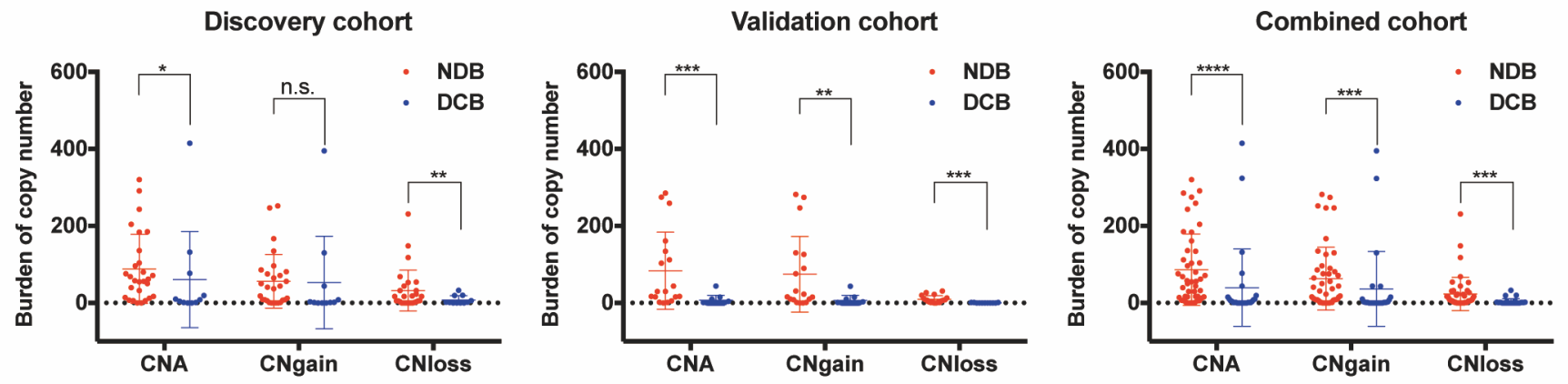

B

CNA in discovery cohort

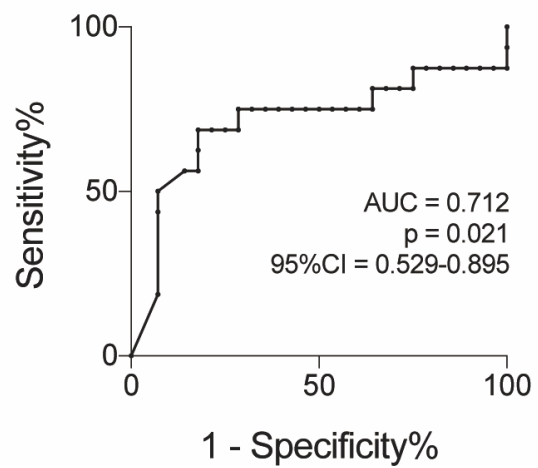

CNA in validation cohort

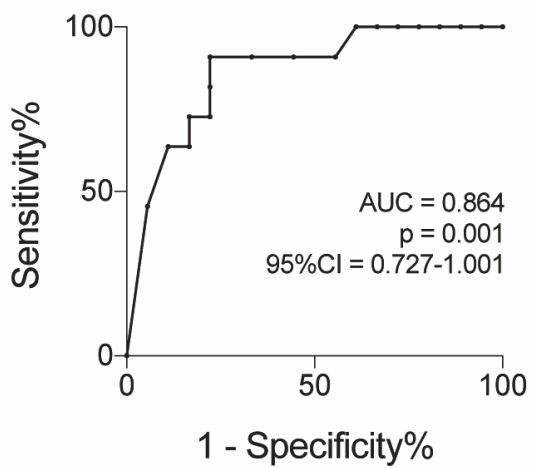

CNA in combined cohort

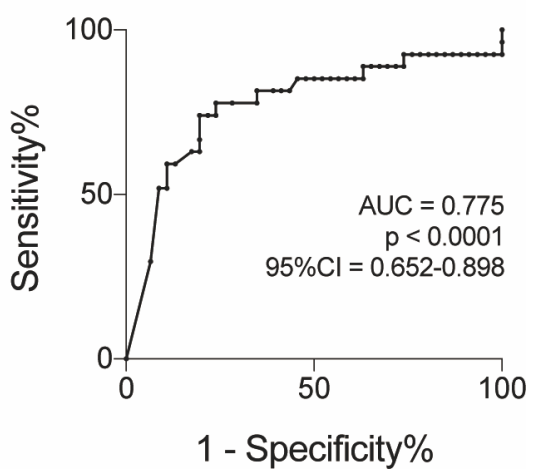

Figure 1 The burden of tumor copy-number alteration and clinical benefit in the Gl cancer cohort. (A) The correlation of burden of copy-number changes (CNA, CNgain and CNloss) and clinical benefit in the discovery $(n=44)$, validation $(n=29)$, and combined $\mathrm{Gl}$ cancer cohort $(\mathrm{n}=73)$. (B) ROC curves for prediction of DCB by CNA burden in the discovery, validation, and combined Gl cancer cohort. DCB, durable clinical benefit; Gl, gastrointestinal; NDB, no durable benefit; ROC, receiver operating characteristic curve; AUC, area under curve.

\section{Burden of CNA predicts response to ICB in the GI cancer cohort}

Tumor somatic copy-number changes have recently been associated with the clinical response to ICB in melanoma. ${ }^{811}$ We therefore investigated the predictive value of the three indices: CNA burden, CNgain, and CNloss in the GI cancer cohort $(n=73)$, which was randomly assigned into the discovery and validation cohorts. First, we observed no remarkable differences in the number of copy-number changes among different GI cancer types (online supplementary figure S1 A-C).

Next, we identified that the CNA burden index revealed significantly decreased levels in DCB versus NDB patients receiving immunotherapy in the GI cohort, with AUCs of 0.712 (discovery, $p=0.021$ ), 0.864 (validation, $p=0.001$ ), and 0.775 (the combined GI cancer patients, $\mathrm{p}<0.0001$ ) (figure 1A,B). Accordingly, a trend toward a lower burden of CNgain or CNloss was also observed in DCB group compared with NDB group with an AUC value of 0.728 (all GI cancer patients, $\mathrm{p}=0.001$ ) and 0.757 (all GI cancer patients, $\mathrm{p}=0.0003$ ) for CNgain and CNloss, respectively (online supplementary figure S2 A-B). Notably, the change in CNgain between DCB and NDB patients did not attain statistical significance in the discovery cohort (online supplementary figure S2 A).

Moreover, efficacy was further compared between groups with high $(\mathrm{CNA}>10)$ and low $(\mathrm{CNA} \leq 10)$ copynumber variation. Specifically, a drastically improved DCB rate was observed in CNA-low (69\%) GI cancers compared with CNA-high (15.9\%) cases (online supplementary table S2, $\mathrm{p}<0.001)$. A similar trend of responsiveness was identified in the CNgain-low $(57.1 \%)$ and CNloss-low (57.5\%) groups when compared with the CNgain-high (18.4\%) and CNloss-high $(12.1 \%)$ cases, respectively $(p<0.01$ for all comparisons, online supplementary table S2). Taken together, our findings strongly indicate that features for tumor genome alterations, including total CNA, CNgain, and CNloss, are potential predictive biomarkers for GI cancers with ICB treatment. Notably, the CNA burden demonstrated the highest AUC value, and therefore, the utility of the CNA burden was further evaluated. 
A

Discovery cohort

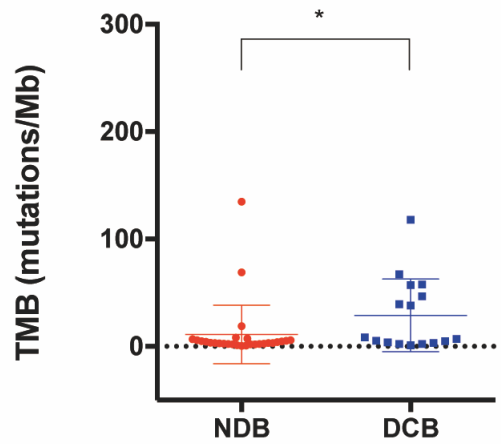

B Discovery cohort

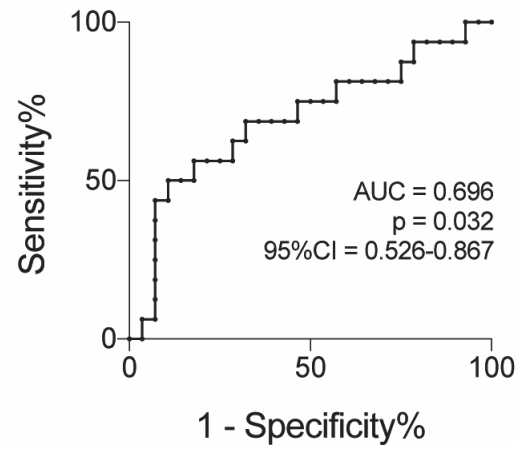

Validation cohort

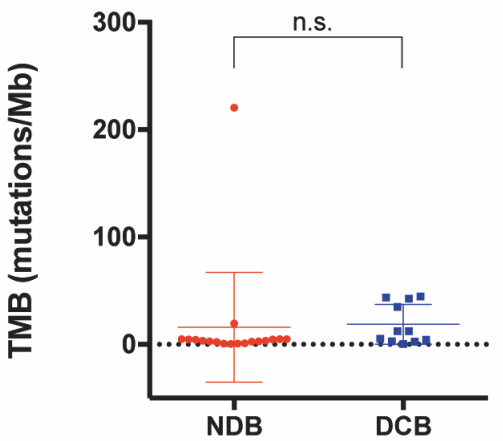

Validation cohort

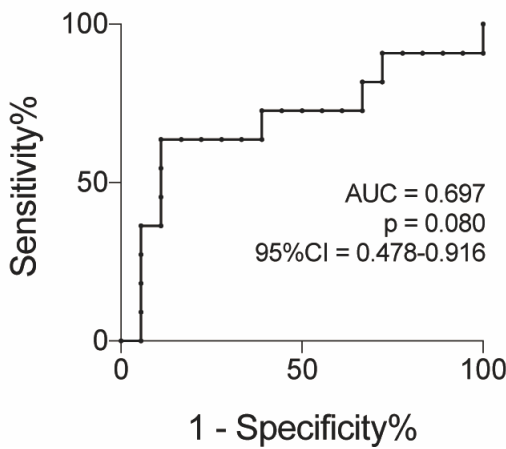

Combined cohort

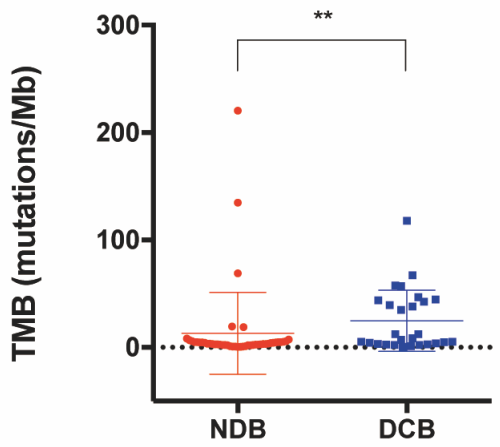

Combined cohort

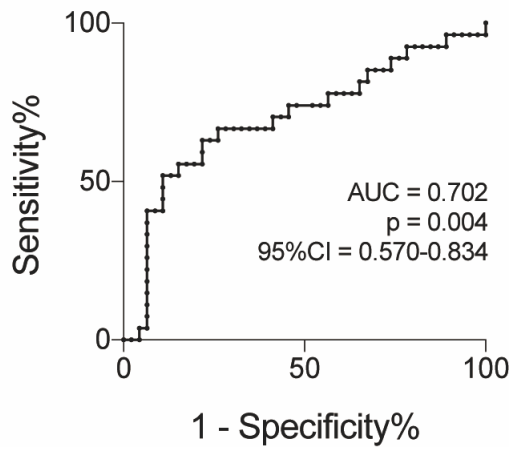

Figure 2 Tumor mutational burden (TMB) and clinical benefit. (A) The correlation of TMB (number of non-synonymous mutations per $\mathrm{Mb}$ ) and clinical benefit in the discovery, validation, and combined $\mathrm{Gl}$ cancer cohort. (B) ROC curves for prediction of DCB by TMB in the discovery, validation, and combined Gl cancer cohort. Gl, gastrointestinal.

\section{The evaluation of predictive value of MSI/MMR and PD-L1 in GI cancers}

Since MSI/MMR and PD-L1 expression have emerged as potential predictive biomarkers for PD-1/PD-L1 blockade, we examined the correlation between MSI/MMR or PD-L1 and the clinical benefit in GI cancer patients. Our data show that MSI-H/dMMR patients experienced a significantly higher DCB rate $(59.1 \%)$ than MSI-L/MSS/ pMMR patients $(28.6 \%, \mathrm{p}=0.022)$, while no association was found between PD-L1 positivity and the efficacy of ICB therapy in our cohort (online supplementary table S2).

\section{Analysis of the predictive value of TMB in GI cancers}

As shown in online supplementary figure S1D, higher TMB was identified in CRC (median 38.7 mutations/ $\mathrm{Mb})$ than other cancer types (median 3.5 mutations/ $\mathrm{Mb}$ ). The median number of TMB in DCB patients was significantly higher than that of NDB patients, with AUCs of $0.696(\mathrm{p}=0.032)$ and $0.702(\mathrm{p}=0.004)$ in the discovery and combined cohorts, respectively (figure 2A,B). On the other hand, we did not observe a statistically significant AUC value (0.697) of TMB in the validation cohort $(\mathrm{p}=0.080)$ (figure 2B). In all GI cancer patients, 60\% $(18 / 30)$ of the patients with a higher mutation burden (designated as above 5, including intermediate and high TMB levels ${ }^{23}$ ) experienced a DCB compared with $20.9 \%$ $(9 / 43)$ of those with a lower TMB (online supplementary table $\mathrm{S} 2, \mathrm{p}=0.001$ ).

\section{Clinical outcomes stratified by baseline CNA, TMB, MSI/MMR} and PD-L1 status in GI cancers

To assess whether the above-tested signatures, including CNA, TMB, MSI/MMR and PD-L1 status, influence the OS and progression-free survival (PFS), we used KaplanMeier analysis for the GI cancer cohort (figures 3 and 4 and online supplementary figure S3-4). As expected, CNA-low patients demonstrated a longer median OS (unreached for all three cohorts) than the CNA-high group (discovery, 7.63 months; validation, 5.6 months; and combined cohorts, 7.43 months) (figure 3A, logrank test, $\mathrm{p}<0.05$ for all comparisons). A similar trend was identified between CNgain/CNloss and OS in GI cancer patients, although statistical significance was not attained for CNgain in the validation cohort (online supplementary figure S3). In addition, patients with higher TMB levels revealed longer OS than patients with lower TMB in the validation and combined cohorts (figure 3B). In our cohort, neither MSI/MMR nor PD-L1 status was able to predict the OS outcome after ICB (figure 3C,D). 
A

Discovery cohort

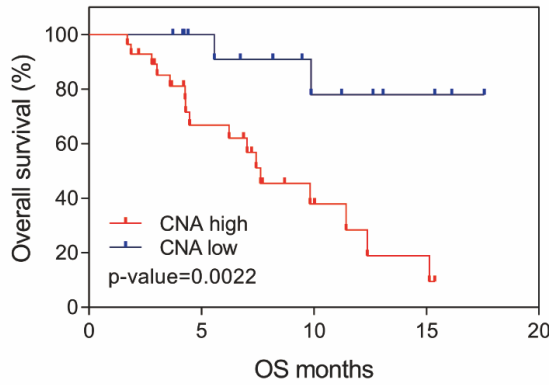

B

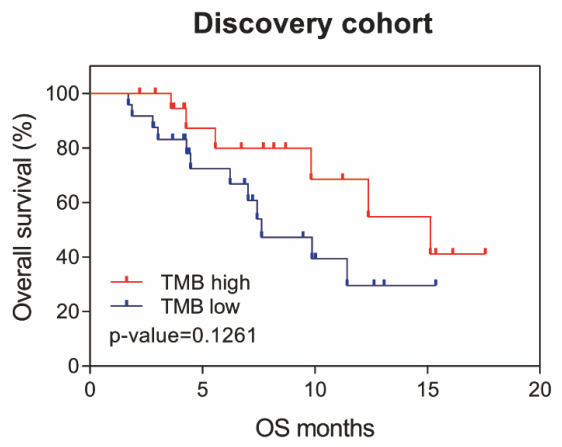

Validation cohort

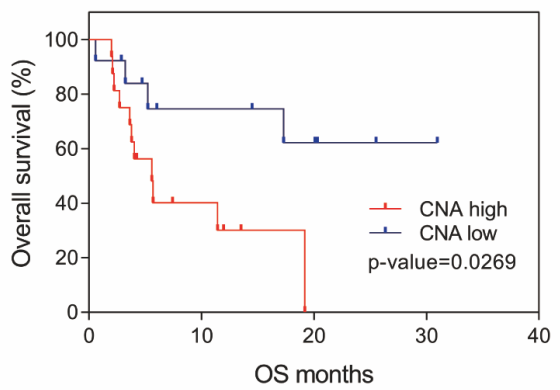

Validation cohort

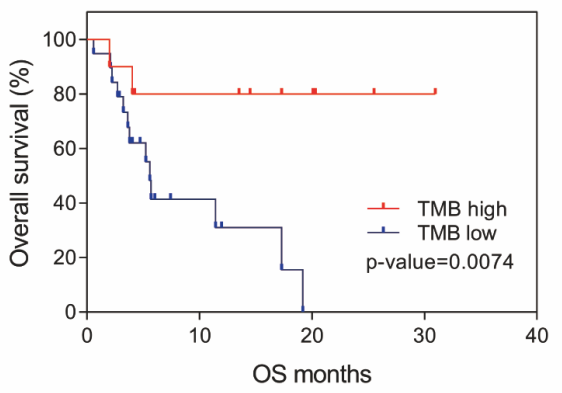

Combined cohort

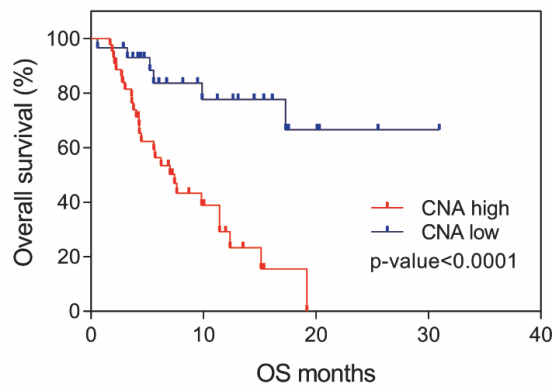

Combined cohort

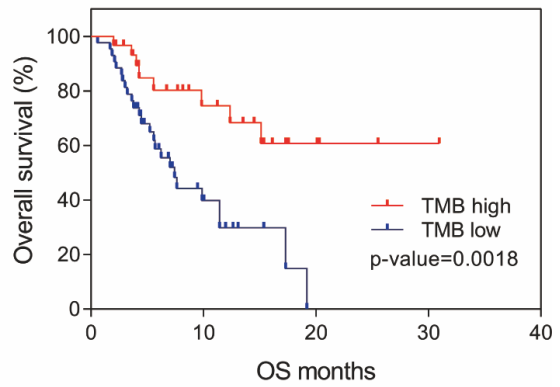

C

D

Combined cohort

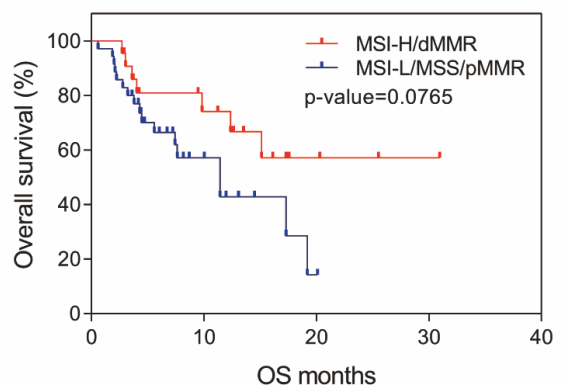

Combined cohort

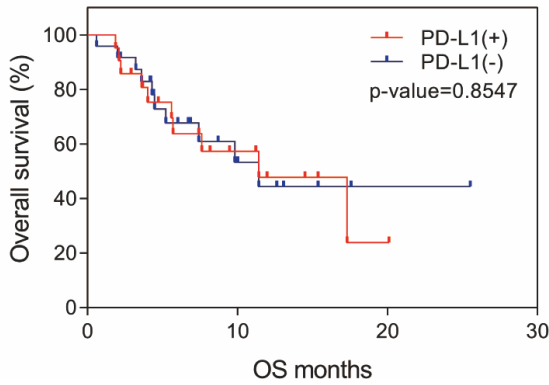

Figure 3 The correlation of tumor CNA burden, TMB level, PD-L1 expression, and MMR/MSI status with the overall survival (OS) of patients with GI cancer receiving ICB treatment. (A) OS (Kaplan-Meier curves) of patients in the discovery, validation, and combined GI cancer cohort, stratified by the levels of CNA burden. (B) OS (Kaplan-Meier curves) of patients in the discovery, validation, and combined Gl cancer cohort, stratified by the levels of TMB. (C) OS (Kaplan-Meier curves) of patients stratified by MSI/MMR status in the combined Gl cancer cohort. (D) OS (Kaplan-Meier curves) of patients stratified by PD-L1 expression in the combined GI cancer cohort. CNA, copy-number alterations; GI, gastrointestinal; ICB, immune-checkpointblockade; MMR, mismatch repair; MSI-H, microsatellite instability high; PD-L1, program death ligand 1; TMB, tumor mutational burden.

Consistently, longer PFS was identified in the lower burden groups for CNA, CNgain or CNloss than in the higher burden groups (figure $4 \mathrm{~A}$ and online supplementary figure $\mathrm{S} 4 \mathrm{~A}-\mathrm{B}, \log$-rank test, $\mathrm{p}<0.05$ for all comparisons, except for CNgain in the validation cohort). In contrast, higher TMB levels were able to stratify patients with favorable PFS in all three cohorts (figure 4B). Moreover, the median PFS time of MSI-H/dMMR patients (7.24 months) was significantly longer than that of MSI-L/MSS/pMMR patients (2.67 months, $\mathrm{p}=0.0173$ ) (figure 4C). However, no significant association was identified between PD-L1 status and PFS in the combined cohort (figure 4D).

\section{Joint utility of burden of CNA with TMB}

Due to the predictive and prognostic value of CNA and $\mathrm{TMB}$, we sought to explore the possibility of combining the two parameters in identifying DCB patients receiving immunotherapy. As revealed in figure 5A, no significant correlation was observed between CNA burden and TMB, 
A

Discovery cohort

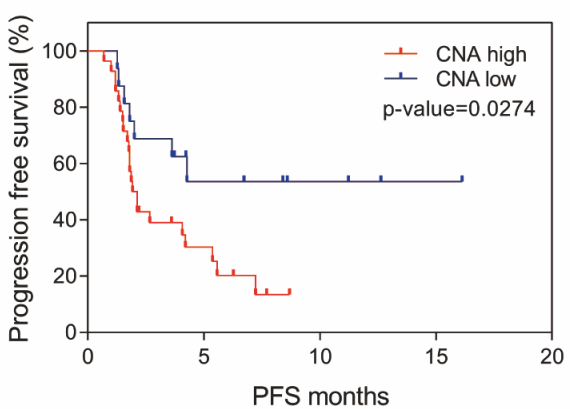

B

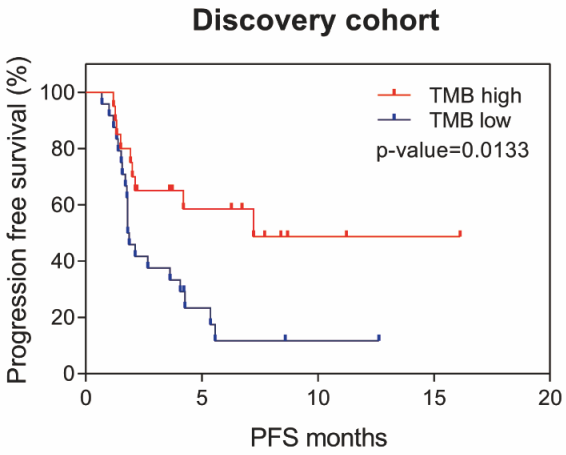

Validation cohort
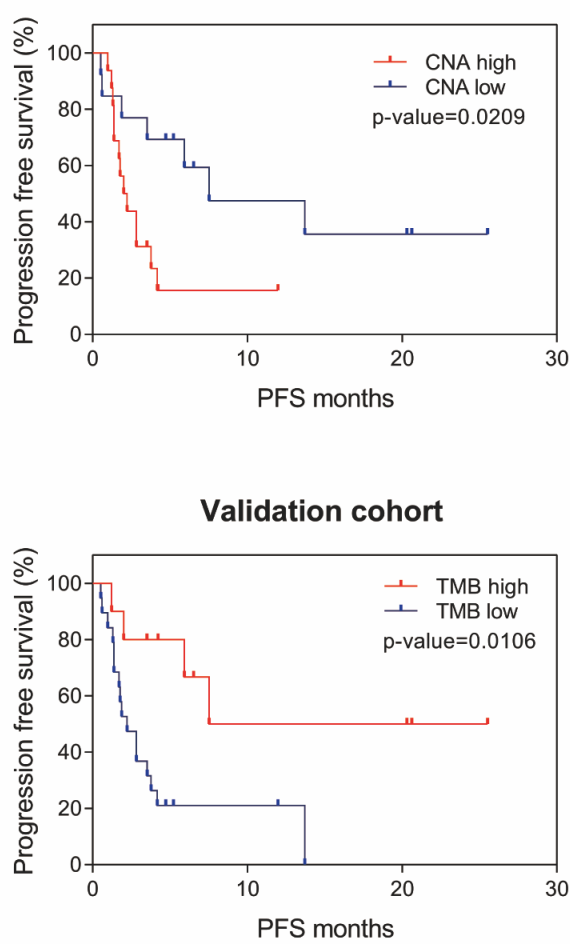

Combined cohort

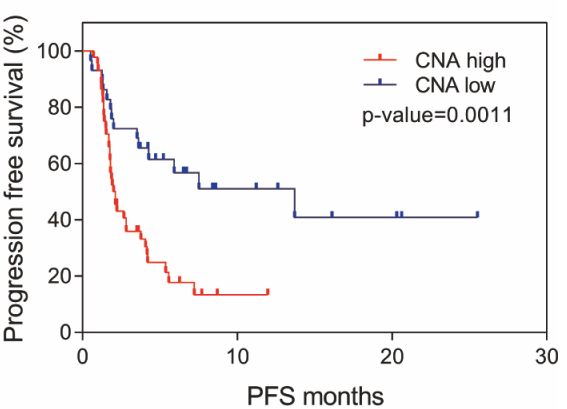

Combined cohort

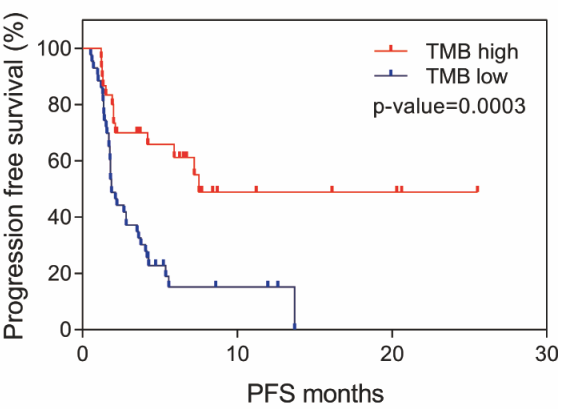

C

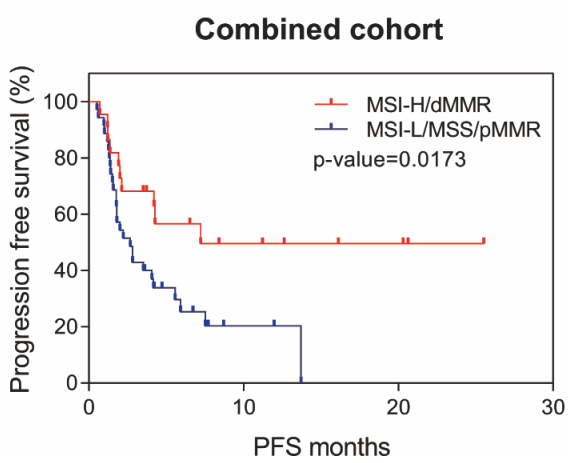

D

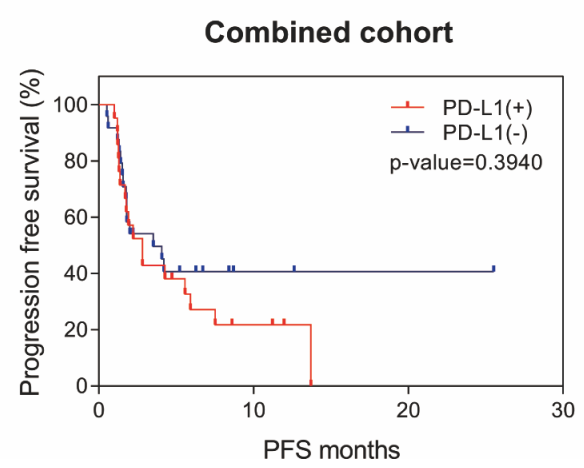

Figure 4 Association between tumor CNA burden, TMB level, PD-L1, and MMR/MSI status with the progression-free survival (PFS) of patients with GI cancer receiving ICB treatment. (A) Kaplan-Meier estimates of PFS according to tumor CNA burden in the discovery cohort, the validation cohort, and the combined Gl cancer cohort. (B) Kaplan-Meier estimates of PFS according to TMB levels in the discovery cohort, the validation cohort, and the combined Gl cancer cohort. (C) Kaplan-Meier estimates of PFS according to MSI/MMR status in the combined Gl cancer cohort. (D) Kaplan-Meier estimates of PFS according to PD-L1 expression in the combined Gl cancer cohort. CNA, copy-number alterations; dMMR, mismatch repair deficient; GI, gastrointestinal; ICB, immune-checkpoint-blockade; MMR, mismatch repair; MSI-H, microsatellite instability high; PD-L1, program death ligand 1; TMB, tumor mutational burden.

suggesting no redundant feature between the two genomic determinants. All 73 patients can be classified into four subgroups based on TMB (high or low) and burden of CNA (high or low), and then the proportion of DCB patients was calculated in each subgroup (figure 5B-E). Notably, the proportion of DCB patients was remarkably higher in the TMB-high/CNA-low subgroup of patients (12 of 14) compared with the TMB-low/CNA-high subgroup (1 of 28) (figure 5C, Fisher's exact test, $\mathrm{p}<0.05$ ). Moreover, the median OS time of the TMB-high/CNA-low subgroup (unreached) was significantly improved compared with the other three subgroups (TMB-low/CNA-low, 17.3 months; TMB-high/CNA-high, 12.37 months; TMB-low/ CNA-high, 6.23 months) (figure $5 \mathrm{D}$, log-rank test $\mathrm{p}<0.05$ for all comparisons).

Moreover, in order to validate the predictive value of CNA burden and its joint utility with TMB, an independent validation was performed using a cohort of 20 GC patients. Consistently, an increased DCB rate was observed in CNAlow (60\%) GC patients compared with CNA-high (0\%) cases (online supplementary figure S5A, Fisher's exact test, $\mathrm{p}<0.01)$. However, there was no significant difference in DCB 
A

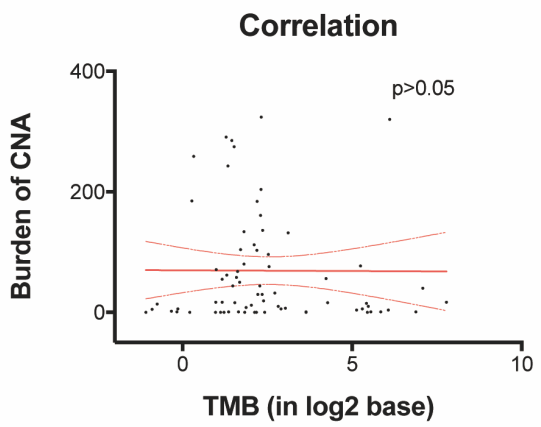

C

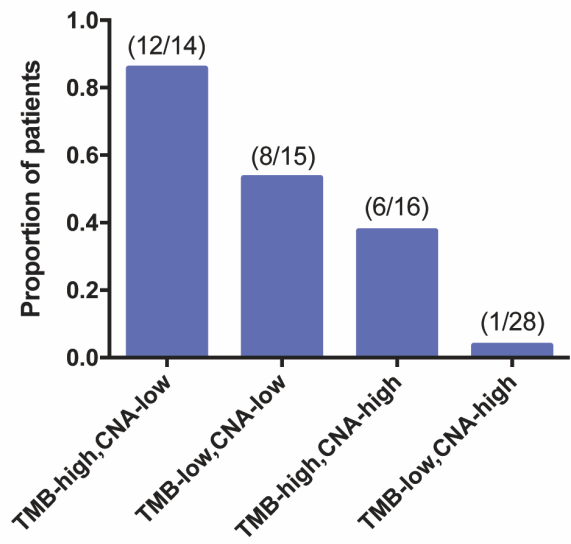

B

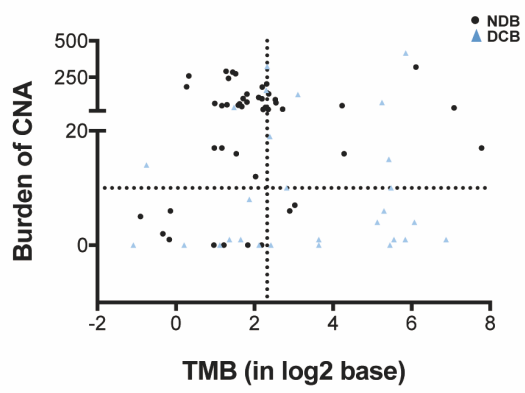

D

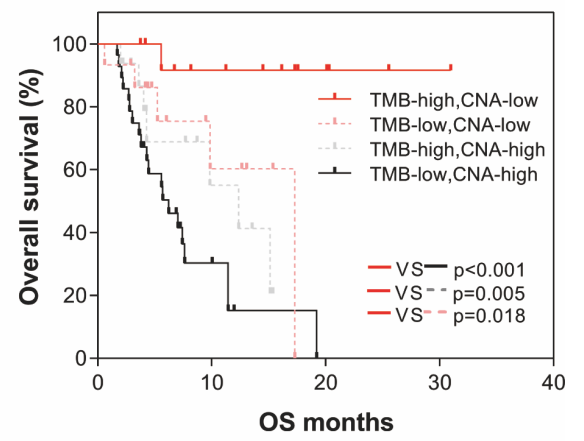

E

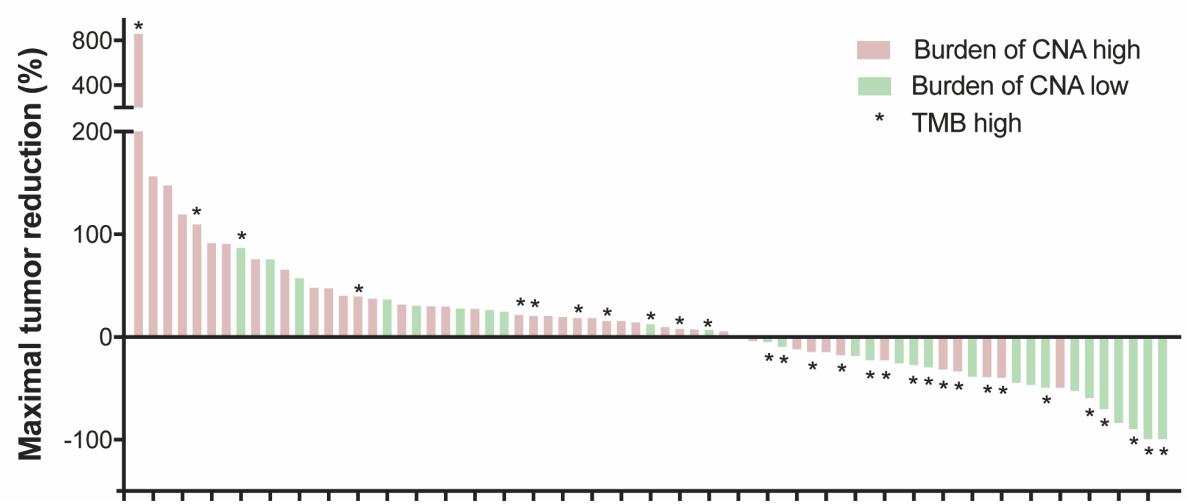

Figure 5 The association of a joint tumor CNA burden/TMB with clinical outcome of patients with Gl cancer receiving ICB treatment. (A) Correlation between the burden of CNA and TMB in the Gl cancer cohort. (B) Relationships of both CNA burden and TMB signatures with clinical benefit (DCB, blue dot; NDB, black dot). (C) Proportions of patients with DCB calculated within each of the four indicated subgroups. (D) Overall survival (OS, Kaplan-Meier curves) of patients within each of the four indicated subgroups in combined Gl cancer cohort. (E) Waterfall plot of tumor response to ICB according to the CNA burden (CNA-high, red bar; CNA-low, green bar) and TMB (asterisk). The Y-axis represents the percentage of maximum tumor reduction assessed according to the RECIST V.1.1 criteria. CNA, copy-number alterations; DCB, durable clinical benefit; Gl, gastrointestinal; ICB, immune-checkpoint-blockade; NDB, no durable benefit; RECIST, Response Evaluation Criteria in Solid Tumors; TMB, tumor mutational burden.

rates between TMB-high and TMB-low subgroups (online supplementary figure S5B). Intriguingly, a high proportion of DCB rate was also identified in the TMB-high/CNA-low subgroup of patients (five of six), as presented in online supplementary figure S5C-D. A larger sample size may help improve the performance of the predictive model.

\section{Lower CNA burden correlates with activated immune responses}

Recent analyses have linked cancer genomic features, including TMB and CNA, with antitumor immunity. In particular, studies have proposed that high mutational load and low aneuploidy may correlate with increased T-cell 
IFNgamma signature

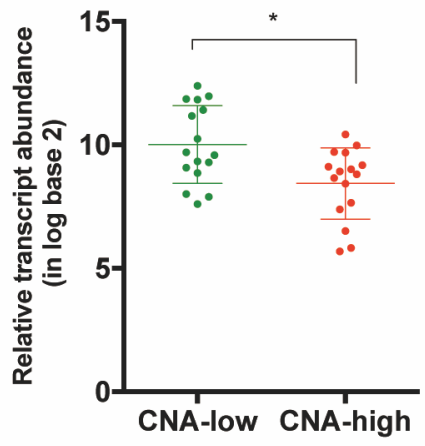

Expanded immune signature

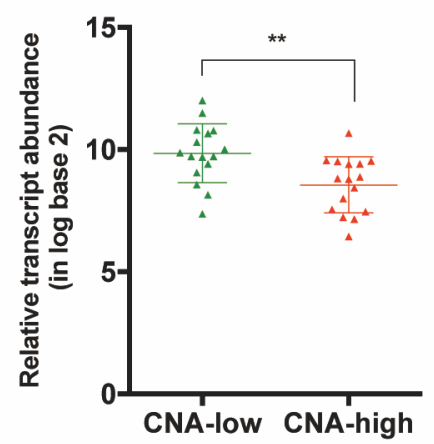

Up-reguated genes CNA low v.s. CNA high

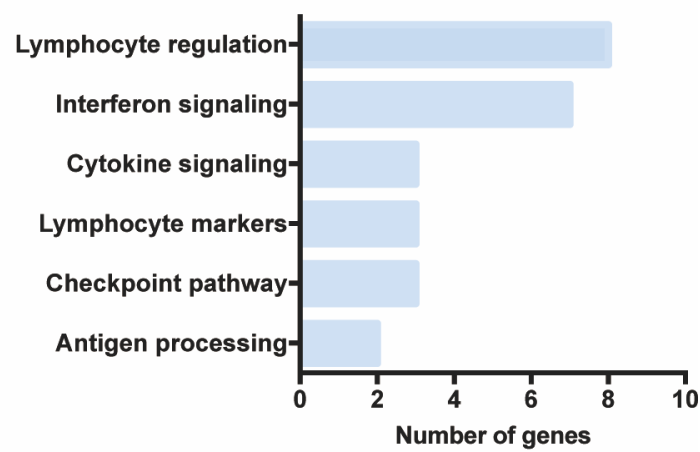

C
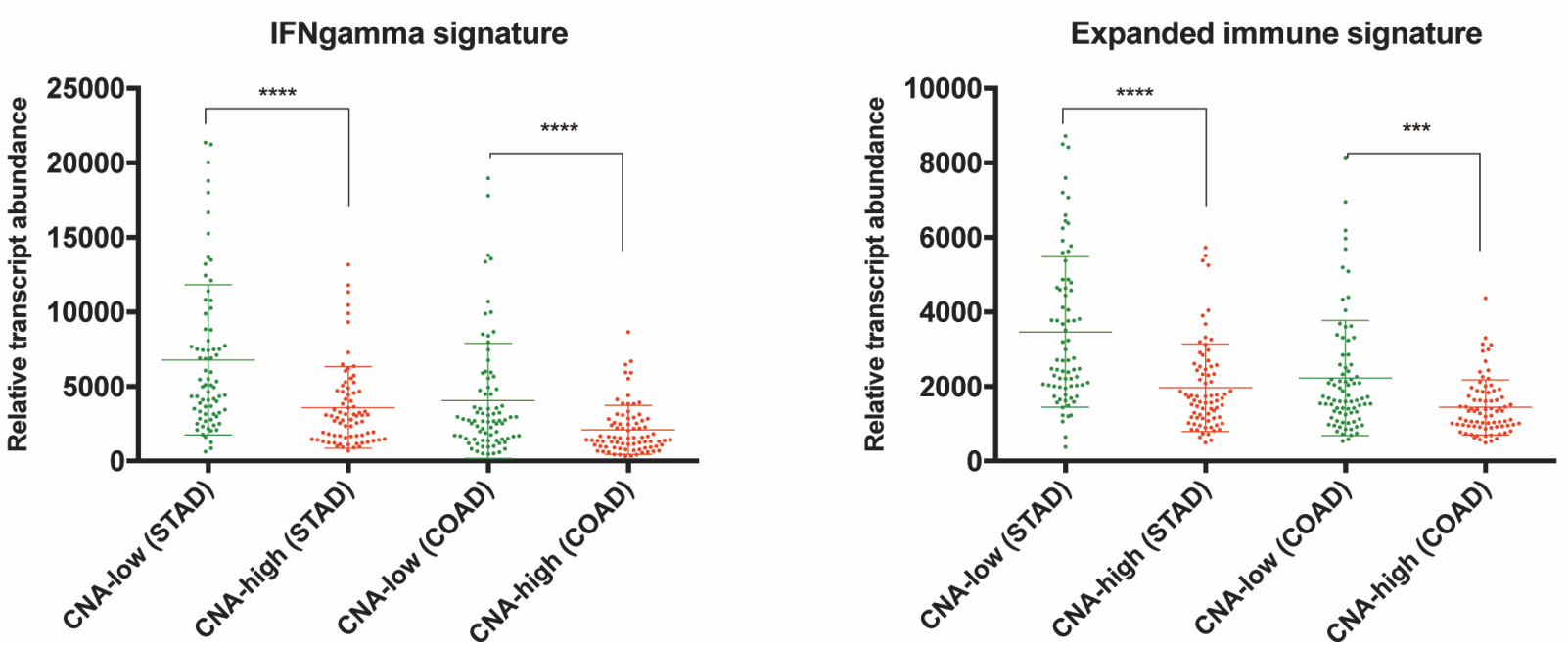

Figure 6 The correlation of the CNA burden and the immune-related RNA signatures. (A) Immune RNA signature levels in CNA-low and CNA-high subgroups in our combined Gl cancer cohort. (B) Upregulated gene clusters in CNA-low vs CNAhigh subgroups in our combined GI cancer cohort. (C) Immune RNA signature levels in CNA-low and CNA-high subgroups in the TCGA STAD and COAD cohorts.CNA-low, tumors with a CNA burden level within the 25th percentile; CNA-high, tumors with a CNA burden level higher than the 75th percentile. CNA, copy-number alterations; COAD, colon adenocarcinoma; GI, gastrointestinal; STAD, stomach adenocarcinoma; TCGA, The Cancer Genome Atlas.

responses. $^{811} 15$ We therefore sought to determine the association between CNA burden and the inflammatory characteristics of the TME by using an RNA IO sequencing platform. IFN- $\gamma$-related (6-gene set) and expanded immune signature (18-gene set) mRNA profiles have recently been developed as pan-cancer biomarkers to predict the immune response to ICB in melanoma, head and neck squamous cell carcinoma and GC cohorts. ${ }^{24}$ Herein, we measured the IFN- $\gamma$ and expanded immune signatures in 65 patient samples with an available RNA data set. Briefly, the CNA burden was partitioned into high or low at the upper quartile or lower quartile value for the 65 GI patients. As figure 6A demonstrates, the CNA-low subgroup revealed higher IFN- $\gamma$ and expanded immune signatures than the CNA-high subgroup $(\mathrm{p}<0.05)$. Moreover, we observed 26 upregulated genes in the CNA-low samples versus the
CNA-high group (figure 6B). These upregulated immunerelated genes belong to the following pathways: lymphocyte markers, interferon signaling, lymphocyte regulation, and checkpoint pathways.

To confirm the correlation between tumor CNA burden and immune signature more broadly, we examined the IFN- $\gamma$ and expanded immune signatures in the TCGA, STAD, and COAD cohorts. For each cohort, the burden of CNA was measured and then partitioned into high or low at the upper quartile or lower quartile value. Consistently, CNA-low samples showed higher immune signatures in both cohorts (figure 6C). Further ssGSEA analysis revealed that CNA-low GC and CRC samples were infiltrated with diverse immune cell types, including activated CD8+ T cells, activated CD4+ T cells, natural killer (NK) cells, and NK T cells (online supplementary figure S6A-B). Collectively, our 
data indicate that a lower CNA burden may correlate with an activated inflammatory response in the TME.

\section{DISCUSSION}

In this study, we used WES analysis to characterize genomic determinants of the clinical benefit from ICB in GI cancers. Here, we show that the CNA burden may have superior predictive value compared with other signatures, including PD-L1, MSI, and TMB.

PD-L1 and MSI/MMR status have been suggested to be independent predictive biomarkers for ICB, and determining MSI/MMR and PD-L1 status of patients based on immunohistochemistry becomes a cost-effective screening tool. ${ }^{25} 26$ In our study, MSI-H/dMMR GI patients showed a significantly increased DCB rate $(59.1 \%)$ versus patients with MSS/MSI-L/pMMR status (28.6\%) (online supplementary table $\mathrm{S} 2, \mathrm{p}=0.022$ ). A larger sample size may increase the predictive value of MSI-H status; however, the overall incidence $(0-5 \%)^{3-5}$ was still too low, and even half of MSI-H/dMMR GI cancers are non-responsive to ICB treatment. ${ }^{27} 28$ On the other hand, we observed that PD-L1 positivity showed little value for stratification of DCB patients with GI cancers (online supplementary table S2). Similarly, in the combination arm of KEYNOTE-062 study, KEYTRUDA plus chemotherapy was not found to be superior for OS (CPS $\geq 1$ or $\mathrm{CPS} \geq 10$ ) or PFS $(\mathrm{CPS} \geq 1)$ compared with chemotherapy alone. ${ }^{29}$ The result confirmed our findings that PD-L1 expression is not a predictive biomarker in advanced gastric or gastroesophageal junction adenocarcinoma. Therefore, we recruited the second validation cohort including 20 GC patients for further investigation.

TMB was recently elevated as a powerful predictive marker for immunotherapy in various cancer types. ${ }^{17233031}$ Here, we identified that a relatively higher level of TMB (>5 mutations/Mb) in GI cancers correlated with improved DCB $(60 \%)$ compared with lower TMB ( $\leq 5$ mutations/Mb, 20.9\%) (online supplementary table S2). We also examined the landscape of TMB across different GI cancer types, revealing that CRC had significantly higher TMB than other malignancies (online supplementary figure S1D). This may result from the high percentage of MSI-H/dMMR CRC patients enrolled in the ICB treatment, and MSI-H/dMMR tumors usually exhibit high TMB, especially in CRC. ${ }^{17} 32$ We can reasonably hypothesize that the predictive power of TMB can partially be attributed to MSI-H/dMMR CRC. Consequently, the best cut-off values for TMB across different GI cancer types still need to be thoroughly explored in larger cohorts in the future. While growing evidence revealed a significant association between TMB and therapeutic efficacy in multiple cancers receiving ICBs, the utility of TMB still remains controversial. ${ }^{2}$ For instance, TMB may function as an effective predictor of response in anti-PD-1 monotherapy in PD-L1-positive lung cancer. ${ }^{33}$ In contrast, in metastatic NSCLC patients receiving pembrolizumab plus platinum-based chemotherapy, TMB did not show robust predictive value. ${ }^{34}$

Most importantly, WES analysis demonstrated a strong correlation between CNA burden and DCB from ICB (figure 1 and online supplementary figure S5A) and that a lower CNA burden was also associated with favorable OS and PFS (figures 3A and 4A). A similar trend was identified in the other two indices, CNgain and CNloss, although statistical significance was not attained in some cohorts (online supplementary figure S2-4). This finding is in accordance with a previous investigation which reported that a higher burden of CNloss is associated with persistent resistance in melanoma patients with sequential CTLA-4 and PD-1 blockades. However, the study did not report significant differences in the CNA burden, CNgain or CNloss in the context of each individual agent response. ${ }^{8}$ Thus, the association between copy-number variations and immune response may vary among different cancer types. Our data strongly indicate that the CNA burden may function as a potential predictive and prognostic biomarker in GI cancers.

Recently, CNA burden has also been correlated with clinical outcome in multiple cancer types. ${ }^{35} 36$ In addition, great efforts have been made to understand how copy-number changes affect the characteristics of cancer and/or the microenvironment. ${ }^{37-40}$ For instance, in hepatocellular carcinoma, a subgroup of patients defined as immune class with inflammatory response showed a lower burden of CNgains and CNlosses; ${ }^{41}$ similarly, pancreatic ductal adenocarcinoma (PDA) with high cytolytic activity exhibited fewer copy-number changes at loci important in PDA, including NOTCH2, MYC, and FGFR1. ${ }^{42}$ A comprehensive analysis of genetic and immunologic characteristics based on the TCGA data set demonstrated a negative correlation between CNA load and immune parameters in COAD and STAD. ${ }^{15}$ This finding was in accordance with our observations that CNA-low samples exhibited higher IFN- $\gamma$ and expanded immune signatures than the CNAhigh subgroup (figure 6). Additionally, ssGSEA analysis further proved that CNA-low GC and CRC samples were infiltrated with activated CD8+ T cells, activated CD4+ $\mathrm{T}$ cells, and NK cells (online supplementary figure S6). Taken together, these results indicate somatic CNA levels might be a strong biomarker for cytotoxic immune cell infiltration and hence predict ICB efficacy.

Finally, we also evaluated the joint utility of CNA burden and TMB (figure 5, and online supplementary figure S5). Intriguingly, a limited DCB (1/28) and OS (6.23 months) from ICB was observed in the TMBlow/CNA-high subgroup, whereas the highest DCB rate $(12 / 14)$ and longest OS (unreached) occurred in the TMB-high/CNA-low subgroup. It has been proposed that higher TMB levels may increase the chances of generating immunogenic neoantigens. ${ }^{43}$ On the other hand, SCNA load, which has been identified as a significant survival predictor independent of TMB in several cancer types, ${ }^{84}$ revealed a significant correlation with immunological features. Specifically, lower levels of CNAs showed 
downregulated expression of cell cycle markers and elevated expression of cytotoxic markers and immune cell infiltration. ${ }^{11}$ Collectively, these findings indicate that using two biomarkers jointly may help to identify potential immunotherapy responders receiving ICB treatment.

Our study has limitations. First, since this cohort was relatively heterogeneous, the proposed signature may vary across GI cancer types or checkpoint inhibitor regimens. Second, this is a retrospective single-center study. Further prospective study within specific cancer type is warranted in the future. In recent years, cost-effective sequencing panels have been developed to accurately measure TMB levels. However, data on the parameters influencing panel-based CNA burden quantification are limited. Finding a refined set of panel tools that can accurately estimate both CNA and mutational burden will become promising for clinical application.

Our data indicate that lower CNA burden correlates with better clinical outcome. Moreover, a joint biomarker of CNA burden and TMB may better stratify DCB patients with GI cancers receiving ICB treatment. Further prospective studies are needed to validate this observation across multiple GI cancer types.

Acknowledgements We acknowledge Zhongwu Li and Yumei Lai for histological analysis with immunohistochemistry staining and Yong Cui and Zhilong Wang for imaging analysis. We thank all of the participating patients, and their families.

Contributors LS and HZ conceived and designed the study. ZL, HC, SL, JZ, and $\mathrm{XJ}$ carried out most of the experimental work and contributed to manuscript writing. SL collected the samples. JG, JL, ZP, XZ, ML, HS, WH, LW, JY, and ZW were involved in acquisition of data and critical revision of the manuscript for important intellectual content. ZL and HZ contributed to the statistical analysis. All authors read and approved the final manuscript.

Funding This work was supported by National Key Research and Development Program of China (2017YFC1308900), Beijing Municipal Science and Technology Commission Program (Z141107002514013), the National Key Sci-Tech Special Project of China (2018ZX10302207), National Key R\&D Program of China (2018YFC1313304), and Digestive Medical Coordinated Development Center of Beijing Hospitals Authority (№. XXT19).

Competing interests None declared.

Patient consent for publication Not required.

Ethics approval This study was approved by the medical ethics committee of Peking University Cancer Hospital.

Provenance and peer review Not commissioned; externally peer reviewed.

Data availability statement Data are available upon reasonable request. Data are available upon reasonable request.

Open access This is an open access article distributed in accordance with the Creative Commons Attribution Non Commercial (CC BY-NC 4.0) license, which permits others to distribute, remix, adapt, build upon this work non-commercially, and license their derivative works on different terms, provided the original work is properly cited, appropriate credit is given, any changes made indicated, and the use is non-commercial. See http://creativecommons.org/licenses/by-nc/4.0/.

\section{ORCID iD}

Lin Shen http://orcid.org/0000-0003-1134-2922

\section{REFERENCES}

1 Kim BJ, Jang HJ, Kim HS, et al. Current status of immune checkpoint inhibitors in gastrointestinal cancers. J Cancer 2017;8:1460-5.
2 Havel JJ, Chowell D, Chan TA. The evolving landscape of biomarkers for checkpoint inhibitor immunotherapy. Nat Rev Cancer 2019;19:133-50.

3 Venderbosch S, Nagtegaal ID, Maughan TS, et al. Mismatch repair status and BRAF mutation status in metastatic colorectal cancer patients: a pooled analysis of the Cairo, CAIRO2, coin, and focus studies. Clin Cancer Res 2014;20:5322-30.

4 Nebot-Bral L, Brandao D, Verlingue L, et al. Hypermutated tumours in the era of immunotherapy: the paradigm of personalised medicine. Eur J Cancer 2017;84:290-303.

$5 \mathrm{Kim}$ J-Y, Shin NR, Kim A, et al. Microsatellite instability status in gastric cancer: a reappraisal of its clinical significance and relationship with mucin phenotypes. Korean J Pathol 2013;47:28-35.

6 Lawrence MS, Stojanov P, Polak P, et al. Mutational heterogeneity in cancer and the search for new cancer-associated genes. Nature 2013;499:214-8.

7 Alexandrov LB, Nik-Zainal S, Wedge DC, et al. Signatures of mutational processes in human cancer. Nature 2013;500:415-21.

8 Roh W, Chen P-L, Reuben A, et al. Integrated molecular analysis of tumor biopsies on sequential CTLA-4 and PD-1 blockade reveals markers of response and resistance. Sci Transl Med 2017;9:eaah3560.

9 Chan TA, Wolchok JD, Snyder A. Genetic basis for clinical response to CTLA-4 blockade in melanoma. N Engl J Med 2015;373:1984.

10 Van Allen EM, Miao D, Schilling B, et al. Genomic correlates of response to CTLA-4 blockade in metastatic melanoma. Science 2015;350:207-11.

11 Davoli T, Uno H, Wooten EC, et al. Tumor aneuploidy correlates with markers of immune evasion and with reduced response to immunotherapy. Science 2017;355. doi:10.1126/science.aaf8399. [Epub ahead of print: 20 Jan 2017].

12 Ock C-Y, Hwang J-E, Keam B, et al. Genomic landscape associated with potential response to anti-CTLA-4 treatment in cancers. Nat Commun 2017;8:1050.

13 Cristescu R, Mogg R, Ayers M, et al. Pan-tumor genomic biomarkers for PD-1 checkpoint blockade-based immunotherapy. Science 2018;362. doi:10.1126/science.aar3593. [Epub ahead of print: 12 Oct 2018].

$14 \mathrm{Kim} \mathrm{HS}$, Cha H, Kim J, et al. Genomic scoring to determine clinical benefit of immunotherapy by targeted sequencing. Eur $J$ Cancer 2019:120:65-74.

15 Budczies J, Seidel A, Christopoulos P, et al. Integrated analysis of the immunological and genetic status in and across cancer types: impact of mutational signatures beyond tumor mutational burden. Oncoimmunology 2018;7:e1526613.

16 Rizvi NA, Hellmann MD, Snyder A, et al. Cancer immunology. mutational landscape determines sensitivity to PD-1 blockade in non-small cell lung cancer. Science 2015;348:124-8.

17 Chalmers ZR, Connelly CF, Fabrizio D, et al. Analysis of 100,000 human cancer genomes reveals the landscape of tumor mutational burden. Genome Med 2017;9:34.

18 Li J, Lupat R, Amarasinghe KC, et al. Contra: copy number analysis for targeted resequencing. Bioinformatics 2012;28:1307-13.

19 Paluch BE, Glenn ST, Conroy JM, et al. Robust detection of immune transcripts in FFPE samples using targeted RNA sequencing. Oncotarget 2017;8:3197-205.

20 Subramanian A, Tamayo P, Mootha VK, et al. Gene set enrichment analysis: a knowledge-based approach for interpreting genome-wide expression profiles. Proc Natl Acad Sci U S A 2005;102:15545-50.

21 Barbie DA, Tamayo P, Boehm JS, et al. Systematic RNA interference reveals that oncogenic KRas-driven cancers require TBK1. Nature 2009;462:108-12.

22 Jia Q, Wu W, Wang Y, et al. Local mutational diversity drives intratumoral immune heterogeneity in non-small cell lung cancer. Nat Commun 2018:9:5361.

23 Goodman AM, Kato S, Bazhenova L, et al. Tumor mutational burden as an independent predictor of response to immunotherapy in diverse cancers. Mol Cancer Ther 2017;16:2598-608.

24 Ayers M, Lunceford J, Nebozhyn M, et al. IFN- $\gamma$-related mRNA profile predicts clinical response to PD-1 blockade. J Clin Invest 2017;127:2930-40.

25 Fehrenbacher L, Spira A, Ballinger M, et al. Atezolizumab versus docetaxel for patients with previously treated non-small-cell lung cancer (poplar): a multicentre, open-label, phase 2 randomised controlled trial. Lancet 2016;387:1837-46.

26 Le DT, Durham JN, Smith KN, et al. Mismatch repair deficiency predicts response of solid tumors to PD-1 blockade. Science 2017;357:409-13.

27 Bupathi M, Wu C. Biomarkers for immune therapy in colorectal cancer: mismatch-repair deficiency and others. J Gastrointest Oncol 2016;7:713-20. 
28 Le DT, Uram JN, Wang H, et al. Pd-1 blockade in tumors with mismatch-repair deficiency. N Engl J Med 2015;372:2509-20.

29 Tabernero J, Cutsem EV, Bang Y-J, et al. KEYNOTE-062: phase III study of pembrolizumab (MK-3475) alone or in combination with chemotherapy versus chemotherapy alone as first-line therapy for advanced gastric or gastroesophageal junction (GEJ) adenocarcinoma. Journal of Clinical Oncology 2019

30 Carbone DP, Reck M, Paz-Ares L, et al. First-Line nivolumab in stage IV or recurrent non-small-cell lung cancer. $N$ Engl J Med 2017;376:2415-26.

31 Hellmann MD, Ciuleanu T-E, Pluzanski A, et al. Nivolumab plus ipilimumab in lung cancer with a high tumor mutational burden. $N$ Engl J Med 2018;378:2093-104.

32 Vanderwalde A, Spetzler D, Xiao N, et al. Microsatellite instability status determined by next-generation sequencing and compared with PD-L1 and tumor mutational burden in 11,348 patients. Cancer Med 2018;7:746-56.

33 Herbst RS, Lopes G, Kowalski DM, et al. Association between tissue TMB (tTMB) and clinical outcomes with pembrolizumab monotherapy (pembro) in PD-L1-positive advanced NSCLC in the KEYNOTE-010 and -042 trials. Annals of Oncology 2019;30:v916-7.

34 Paz-Ares L, Langer CJ, Novello S, et al. Pembrolizumab (pembro) plus platinum-based chemotherapy (chemo) for metastatic NSCLC: tissue TMB (tTMB) and outcomes in KEYNOTE-021, 189, and 407. Annals of Oncology 2019;30:v917-8.

35 Hieronymus $\mathrm{H}$, Murali R, Tin A, et al. Tumor copy number alteration burden is a pan-cancer prognostic factor associated with recurrence and death. Elife 2018;7. doi:10.7554/eLife.37294. [Epub ahead of print: 04 Sep 2018].

36 Zhang L, Feizi N, Chi C, et al. Association analysis of somatic copy number alteration burden with breast cancer survival. Front Genet 2018;9:421.

37 Williams JL, Greer PA, Squire JA. Recurrent copy number alterations in prostate cancer: an in silico meta-analysis of publicly available genomic data. Cancer Genet 2014;207:474-88.

38 Taylor BS, Schultz N, Hieronymus H, et al. Integrative genomic profiling of human prostate cancer. Cancer Cell 2010;18:11-22.

39 Solimini NL, Xu Q, Mermel CH, et al. Recurrent hemizygous deletions in cancers may optimize proliferative potential. Science 2012;337:104-9.

40 Hieronymus H, Schultz N, Gopalan A, et al. Copy number alteration burden predicts prostate cancer relapse. Proc Natl Acad Sci U S A 2014;111:11139-44.

41 Sia D, Jiao Y, Martinez-Quetglas I, et al. Identification of an Immunespecific class of hepatocellular carcinoma, based on molecular features. Gastroenterology 2017;153:812-26.

42 Balli D, Rech AJ, Stanger BZ, et al. Immune cytolytic activity Stratifies molecular subsets of human pancreatic cancer. Clin Cancer Res 2017;23:3129-38.

43 McGranahan N, Furness AJS, Rosenthal R, et al. Clonal neoantigens elicit $\mathrm{T}$ cell immunoreactivity and sensitivity to immune checkpoint blockade. Science 2016;351:1463-9.

44 Liu L, Bai X, Wang J, et al. Combination of TMB and CNA Stratifies prognostic and predictive responses to immunotherapy across metastatic cancer. Clin Cancer Res 2019;25:7413-23. 